\title{
L’accélérateur d'électrons picoseconde ELYSE à Orsay
}

\author{
J. Belloni, M. Gaillard, H. Monard, M. Mostafavi, I. Lampre, H. Remita, \\ J.L. Marignier, J.C. Bourdon ${ }^{1}$ et T. Garvey ${ }^{1}$ \\ Université Paris-Sud, Laboratoire de Chimie Physique, bâtiment 349, 91405 Orsay, France \\ ${ }^{1}$ Laboratoire de l'Accélérateur Linéaire, bâtiment 200, 91405 Orsay, France
}

\begin{abstract}
Résumé. Avec le projet ELYSE, un groupe de physico-chimistes de l'Université d'Orsay Paris-Sud a proposé à la communauté scientifique française de se doter d'une nouvelle plate-forme permettant des études de cinétiques rapides au moyen des techniques complémentaires de radiolyse pulsée et de photolyse laser. L'accélérateur d'électrons, de type photodéclenché, est prévu pour délivrer des impulsions d'électrons de moins de 5 picosecondes avec une énergie comprise entre 5 et $9 \mathrm{MeV}$. Les photoelectrons sont générés au moyen d'un laser picoseconde synchronisé. On décrit les différentes performances de l'appareil, après l'obtention du premier faisceau d'électrons le 15 mars 2002, ainsi que les applications envisagées, plus particulièrement en radiolyse pulsée.
\end{abstract}

\section{INTRODUCTION}

Le Centre de Cinétique Rapide ELYSE, du Laboratoire de Chimie Physique d'Orsay (UMR 8000), résulte de la volonté de physico-chimistes, de biophysiciens et d'opticiens d'Orsay et des environs, de faire progresser leurs recherches par l'étude de la dynamique des systèmes excités par une impulsion très courte d'électrons ou de photons. L'étude des actes chimiques et biochimiques élémentaires constitue un pan de la "science aux temps ultracourts" en plein essor en France et dans le monde.

La technique de choix pour l'observation des phénomènes aux temps très courts est celle dite de "pompe-sonde" qui utilise deux impulsions parfaitement synchronisées. La première ("pompe") initie la réaction à étudier. La seconde ("sonde") beaucoup moins intense et décalée temporellement de la première, sert seulement à analyser le système après excitation. Les variations d'intensité de la seconde, mesurées en fonction du temps, renseignent sur le chemin de réaction suivi par le système et fournissent, en quelque sorte, le film de la réaction.

S'il existe de nombreuses installations au monde qui permettent d'exciter un milieu au moyen d'impulsions de photons ultracourtes (picoseconde, femtoseconde) produites par des lasers, rares sont celles qui offrent des impulsions d'électrons d'une durée inférieure à la nanoseconde.

Le centre ELYSE se caractérise donc par la possibilité d'initier les phénomènes réactifs soit au moyen d'impulsions de l'ordre de la picoseconde, d'électrons ayant une énergie allant de 5 à $9 \mathrm{MeV}$ (radiolyse) soit d'impulsions de photons d'une durée supérieure à 160 
femtosecondes (photolyse). Les deux types d'expériences sont complémentaires car, dans chacun des cas, l'énergie n'est pas déposée de la même façon dans la matière, et les espèces réactives produites sont souvent différentes.

\section{CARACTERISTIQUES DE L'ACCELERATEUR ELYSE}

\subsection{Le laser}

Le laser femtoseconde est un équipement de la société Spectra-Physics qui a plusieurs fonctions. Il forme les électrons par excitation de la photocathode située avant la section accélératrice de l'accélérateur.

Il sert de source de photons impulsionnelle femtoseconde pour les expériences de photophysique et de photochimie.

Enfin il sert de sonde pour les expériences en absorption optique transitoire, tant en radiolyse qu'en photolyse, puisque par sa conception l'ensemble laser-accélérateur sera synchronisé.

\subsection{L'accélérateur d'électrons}

La machine choisie pour équiper ce centre est un accélérateur d'électrons d'énergie de 5 à $9 \mathrm{MeV}$ dont le faisceau est fourni par un canon HF photodéclenché par un laser.

Cette machine doit fournir aux utilisateurs des impulsions d'électrons très courtes (quelques picosecondes) avec une fréquence de répétition supérieure ou égale à $50 \mathrm{~Hz}$.

Le faisceau d'électrons est photodéclenché par un faisceau laser, dont la largeur à mihauteur des impulsions est $\cdot 2 \mathrm{ps}$ avec une énergie supérieure à 70 microJoules à $266 \mathrm{~nm}$. La photocathode qui génère les électrons est constituée d'un alliage de Césium et de Tellure préparé in situ par co-évaporation des deux métaux dans une chambre de préparation située à l'arrière de l'accélérateur. Le cahier des charges des caractéristiques du faisceau de l'accélérateur à électrons est le suivant :

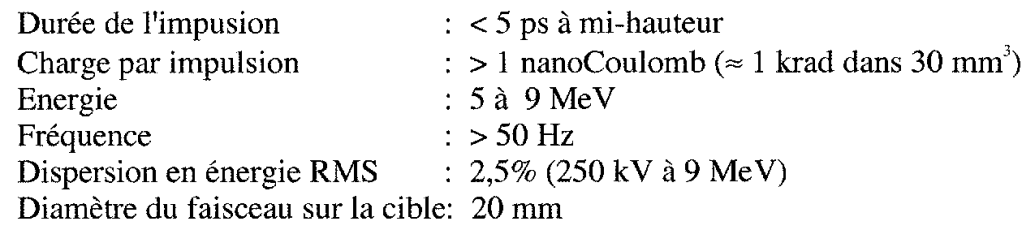

L'une des spécificités d'ELYSE tient dans la possibilité d'effectuer à l'échelle de la picoseconde, des expériences "pompe-sonde" avec une source d'électrons synchronisée avec celle des photons puisque c'est un laser, génèrant des impulsions optiques ultracourtes, qui arrachent les électrons de la photocathode de l'accélérateur. Des paquets d'électrons sont ainsi engendrées, avec une résolution temporelle de l'ordre de la picoseconde, d'où un gain en résolution de plusieurs ordres de grandeur, en comparaison avec les montages de radiolyse pulsée les plus performants. Cet instrument est unique en Europe : seuls deux autres équipements de ce type existent depuis peu dans le monde à Brookhaven aux USA et à Tokaï-Mura au Japon. 


\section{QUELQUES PROJETS SCIENTIFIQUES}

Le montage de radiolyse pulsée est prévu en premier lieu pour étudier les réactions en milieu liquide: étude des effets du rayonnement, utilisation du rayonnement comme source de radicaux libres pour étudier leurs propriétés physiques, chimiques ou biochimiques. Citons, parmi les nombreux projets, l'étude de la dynamique de solvatation d'anions en phase liquide, l'étude du mécanisme de formation et réactivité du réactif de Grignard, l'étude d'agrégats métalliques en milieux confinés. Le montage d'absorption d'espèces transitoires installé sur le laser fait également l'objet de nombreux projets dans lesquels plusieurs équipes d'Orsay et des environs (notamment de l'ENS de Cachan) sont impliquées. Mentionnons : la cinétique de recombinaison de paires électron-trou photoinduites dans des semiconducteurs, l'étude des mécanismes de coupure et de dégradation de l'ADN, la photophysique de composés de coordination moléculaires et supramoléculaires.

\section{QUELLES APPLICATIONS PEUT-ON ENVISAGER POUR CES RECHERCHES ?}

On peut prédire sans grand risque que les recherches menées au centre ELYSE auront des retombées dans des domaines appliqués, certaines des équipes installées à ELYSE ayant une longue expérience de recherches conduites en collaboration avec des partenaires industriels. Rappelons les recherches menées très récemment à Orsay par radiolyse pulsée qui ont permis d'obtenir le décuplement du rendement de formation de l'image latente photographique grâce au un dopage des émulsions par l'ion formiate. Citons également la mise en évidence récente des propriétés de limitation optique de suspensions d'agrégats métalliques synthétisés par voie radiolytique, système susceptible d'être utilisé pour la protection oculaire et des systèmes optroniques face à des impulsions laser intenses.

L'université Paris-Sud pourra s'appuyer sur les chercheurs et enseignants-chercheurs des équipes du centre de cinétique rapide pour participer aux enseignements dans les filières spécialisées (DEA et DESS) dans les domaines de la physico-chimie moléculaire, des radioéléments, rayonnements et radiochimie, de l'optique, optronique et photonique, etc....

ELYSE constitue un centre de recherche fondamentale en cinétique rapide unique en Europe. Les projets de recherche couvrent d'emblée un champ scientifique qui va de la physique moléculaire à la biophysique. Dès son démarrage, ELYSE est un centre à vocation fédérative, ouvert le plus largement possible, en particulier sur toute la Physico-Chimie d'Orsay, mais également sur la communauté environnante et sur des équipes françaises et internationales dès que l'infrastructure le permettra.

Le Centre de Cinétique Rapide ÉLYSE, du Laboratoire de Chimie Physique d'Orsay (UMR 8000), a été inauguré le 6 Juin 2001. Il est situé dans un ancien bâtiment aujourd'hui réhabilité qui abritait un "accélérateur vertical". À l'intérieur des murs ont été aménagés environ $1000 \mathrm{~m}^{2}$ d'aires expérimentales sur 4 niveaux, dont les $3 / 5$ ème sont pour l'instant occupés par le projet ELYSE. Une nouvelle aile a été construite, qui comprend une salle de conférence et 450 $\mathrm{m}^{2}$ de bureaux distribués sur 3 étages. Le chantier commencé le 15 janvier 2000, s'est achevé le 24 Janvier 2001. 